\title{
Applications of Contractive-like Mapping Principles to Fuzzy Equations
}

\author{
Juan J. Nieto and Rosana RodRíGuez-LóPEZ \\ Departamento de Análisis Matemático \\ Facultad de Matemáticas \\ Universidad de Santiago de Compostela, \\ 15782 Santiago de Compostela - Spain \\ amnieto@usc.es amrosana@usc.es
}

Received: January 30, 2006

Accepted: March 31, 2006

\begin{abstract}
We recall a recent extension of the classical Banach fixed point theorem to partially ordered sets and justify its applicability to the study of the existence and uniqueness of solution for fuzzy and fuzzy differential equations. To this purpose, we analyze the validity of some properties relative to sequences of fuzzy sets and fuzzy functions.
\end{abstract}

Key words: fixed points, fuzzy sets, fuzzy equations, fuzzy differential equations, upper and lower solutions, existence and uniqueness of solution.

2000 Mathematics Subject Classification: primary 03E72, 26E50, 03G10; secondary 47H10, 34A12.

\section{Preliminaries}

Results on existence of fixed points in partially ordered sets and some applications to the resolution of matrix equations are presented in [20] and the main result is the following:

Theorem $1.1([20])$. Let $X$ be a partially ordered set such that every pair $x, y \in X$ has an upper and a lower bound. Let $d$ be a metric on $X$ such that $(X, d)$ is complete. Let $f: X \rightarrow X$ be a continuous and monotone (either order-preserving or orderreversing) operator. Suppose that the following assertions hold:

Research supported in part by Ministerio de Educación y Ciencia and FEDER, project MTM2004-06652-C03-01, and by Xunta de Galicia and FEDER, project PGIDIT05PXIC20702PN.

Rev. Mat. Complut. 
- there exists $k \in(0,1)$ with $d(f(x), f(y)) \leq k d(x, y), \forall x \geq y$.

- there exists $x_{0} \in X$ with $x_{0} \leq f\left(x_{0}\right)$ or $x_{0} \geq f\left(x_{0}\right)$.

Then $f$ has a unique fixed point $x^{*} \in X$, and for each $x \in X,\left(f_{n}(x)\right)_{n \in \mathbb{N}} \rightarrow x^{*}$.

In $[16,18]$, the authors analyze the existence of a unique fixed point for mappings defined in partially ordered sets and they show the applicability of the results obtained to the study of the existence of a unique solution for first-order ordinary differential equations with periodic boundary conditions. The authors obtained the following results.

Theorem $1.2([16])$. Let $(X, \leq)$ be a partially ordered set and suppose that there exists a metric $d$ in $X$ such that $(X, d)$ is a complete metric space. Let $f: X \rightarrow X$ be a monotone nondecreasing mapping such that there exists $k \in[0,1)$ with

$$
d(f(x), f(y)) \leq k d(x, y), \forall x \geq y .
$$

Suppose that either $f$ is continuous or $X$ is such that

$$
\text { if a nondecreasing sequence }\left(x_{n}\right)_{n \in \mathbb{N}} \rightarrow x \text { in } X \text {, then } x_{n} \leq x, \forall n \text {. }
$$

If there exists $x_{0} \in X$ with $x_{0} \leq f\left(x_{0}\right)$, then $f$ has a fixed point.

Theorem $1.3([16])$. Let $(X, \leq)$ be a partially ordered set and suppose that there exists a metric $d$ in $X$ such that $(X, d)$ is a complete metric space. Let $f: X \rightarrow X$ be a monotone nondecreasing mapping such that there exists $k \in[0,1)$ with $d(f(x), f(y)) \leq$ $k d(x, y), \forall x \geq y$. Suppose that either $f$ is continuous or $X$ is such that

$$
\text { if a nonincreasing sequence }\left(x_{n}\right)_{n \in \mathbb{N}} \rightarrow x \text { in } X \text {, then } x \leq x_{n}, \forall n \text {. }
$$

If there exists $x_{0} \in X$ with $x_{0} \geq f\left(x_{0}\right)$, then $f$ has a fixed point.

An additional condition which allows to obtain uniqueness of fixed point and global convergence of the method of successive approximations is

every pair of elements of $X$ has a lower bound or an upper bound, or, equivalently,

for every $x, y \in X$, there exists $z \in X$ which is comparable to $x$ and $y$.

For nonincreasing functions, some positive results were presented in [18].

Theorem 1.4 ([18, Theorem 4]). Let $(X, \leq)$ be a partially ordered set satisfying (3) and suppose that there exists a metric $d$ in $X$ such that $(X, d)$ is a complete metric space. Let $f: X \rightarrow X$ be a nonincreasing function such that there exists $k \in[0,1)$ 
with $d(f(x), f(y)) \leq k d(x, y), \forall x \geq y$. Suppose also that either $f$ is continuous or $X$ is such that

$$
\begin{aligned}
\text { if }\left(x_{n}\right)_{n \in \mathbb{N}} \rightarrow & x \text { is a sequence in } X \text { whose consecutive terms are } \\
& \text { comparable, then there exists a subsequence }\left(x_{n_{k}}\right)_{k \in \mathbb{N}} \\
& \text { of }\left(x_{n}\right)_{n \in \mathbb{N}} \text { such that every term is comparable to the limit } x .
\end{aligned}
$$

If there exists $x_{0} \in X$ with $x_{0} \leq f\left(x_{0}\right)$ or $x_{0} \geq f\left(x_{0}\right)$, then $f$ has a unique fixed point.

More generally, for non necessarily monotone operators, it was proved the following theorem.

Theorem 1.5 ([18, Theorem 7$])$. Let $(X, \leq)$ be a partially ordered set and suppose that (3) holds and that there exists a metric $d$ in $X$ such that $(X, d)$ is a complete metric space. Let $f: X \rightarrow X$ be such that $f$ maps comparable elements into comparable elements, that is,

$$
x, y \in X, x \leq y \Longrightarrow\left\{\begin{array}{l}
f(x) \leq f(y) \\
o r \\
f(x) \geq f(y)
\end{array}\right.
$$

and such that there exists $k \in[0,1)$ with $d(f(x), f(y)) \leq k d(x, y), \forall x \geq y$. Suppose that either $f$ is continuous or $X$ is such that condition (4) holds. If there exists $x_{0} \in X$ with $x_{0}$ comparable to $f\left(x_{0}\right)$, then $f$ has a unique fixed point $\bar{x}$. Moreover, $\forall x \in X, \lim _{n \rightarrow+\infty} f^{n}(x)=\bar{x}$.

In this paper, we illustrate the applicability of these results to obtain the existence of a unique solution for a fuzzy equation, that is, an equation defined in the space of fuzzy subsets of $\mathbb{R}^{m}$ and also for fuzzy differential equations.

The paper is organized as follows. In sections 2 and 3, we recall the basic concepts of fuzzy sets and fuzzy functions, presenting some interesting properties for fuzzy spaces. Then we present different orders (section 5) in the space of fuzzy numbers and functions. Finally, we apply the results to the Cauchy problem for fuzzy differential equations (section 6) and to fuzzy functional differential equations with an initial condition (section 7). We obtain new results in the theory of fuzzy differential equations.

\section{Fuzzy sets}

The set of fuzzy real numbers $E^{1}$ is the family of elements $x: \mathbb{R} \longrightarrow[0,1]$ satisfying the following properties [5]:

- $x$ is normal: there exists $t_{0} \in \mathbb{R}$ with $x\left(t_{0}\right)=1$,

- $x$ is upper semicontinuous, 
- $x$ is fuzzy convex,

$$
x\left(\lambda t_{1}+(1-\lambda) t_{2}\right) \geq \min \left\{x\left(t_{1}\right), x\left(t_{2}\right)\right\}, \text { for all } t_{1}, t_{2} \in \mathbb{R}, \lambda \in[0,1],
$$

- The support of $x, \operatorname{supp}(x)=\overline{\{t \in \mathbb{R}: x(t)>0\}}$ is a bounded subset of $\mathbb{R}$.

For a fuzzy number $x \in E^{1}$, we denote the $\alpha$-level set by

$$
[x]^{\alpha}=\{t \in \mathbb{R}: x(t) \geq \alpha\},
$$

which is an interval, since it is a nonempty compact convex subset of $\mathbb{R}$, for every $\alpha \in[0,1]$. Hence $[x]^{\alpha}=\left[x_{\alpha l}, x_{\alpha r}\right]$, for all $\alpha \in(0,1]$, and

$$
[x]^{0}=\overline{\bigcup_{\alpha \in(0,1]}[x]^{\alpha}}=\left[x_{0 l}, x_{0 r}\right] .
$$

In $E^{1}$, we define the following partial orderings: $\leq$ and $\preceq$, given by

$$
x, y \in E^{1}, x \leq y \Longleftrightarrow x_{\alpha l} \leq y_{\alpha l} \text { and } x_{\alpha r} \leq y_{\alpha r} \text {, for all } \alpha \in(0,1],
$$

and

$$
x, y \in E^{1}, x \preceq y \Longleftrightarrow[x]^{\alpha} \subseteq[y]^{\alpha}, \text { for all } \alpha \in(0,1],
$$

that is,

$$
x_{\alpha l} \geq y_{\alpha l} \text { and } x_{\alpha r} \leq y_{\alpha r}, \text { for all } \alpha \in(0,1] .
$$

$E^{1}$ is a complete metric space [5] considering the distance

$$
d_{\infty}(x, y)=\sup _{\alpha \in[0,1]} d_{H}\left([x]^{\alpha},[y]^{\alpha}\right), \text { for } x, y \in E^{1},
$$

where $d_{H}$ represents the Hausdorff distance between nonempty compact convex subsets of $\mathbb{R}$.

For a fuzzy number $x \in E^{1}$, we define the functions

$$
\begin{aligned}
& x_{L}:[0,1] \longrightarrow \mathbb{R}, \quad x_{L}(\alpha)=x_{\alpha l}, \forall \alpha \in[0,1], \\
& x_{R}:[0,1] \longrightarrow \mathbb{R}, \quad x_{R}(\alpha)=x_{\alpha r}, \forall \alpha \in[0,1] .
\end{aligned}
$$

The product and sum of fuzzy numbers $x$ and $y$ is given by the Zadeh's Extension principle: $[x+y]^{\alpha}=[x]^{\alpha}+[y]^{\alpha}$, and $[x \cdot y]^{\alpha}=[x]^{\alpha} \cdot[y]^{\alpha}$, for every $\alpha \in[0,1]$. See [5, page 4$]$ or $[12$, page 3$]$.

We denote by $E^{m}$ the space of all fuzzy subsets $u$ of $\mathbb{R}^{m} u: \mathbb{R}^{m} \rightarrow[0,1]$ such that $u$ is normal, upper semicontinuous, fuzzy convex with bounded support. The level sets of $u$ are obtained similarly to $E^{1}$. Thus, $[u]^{\alpha}$ is a convex and compact subset of $\mathbb{R}^{m}$ (see [5]). 
The supremum metric on $E^{m}$ is defined by

$$
d_{\infty}(x, y)=\sup _{\alpha \in[0,1]} d_{H}\left([x]^{\alpha},[y]^{\alpha}\right), \quad \text { for } x, y \in E^{m}
$$

and $\left(E^{m}, d_{\infty}\right)$ is a complete metric space [5].

For the case of $m$-dimensional fuzzy sets, we can supply $E^{m}$ with a partial ordering $\preceq$ defined similarly to the one-dimensional case:

$$
x, y \in E^{m}, x \preceq y \Longleftrightarrow[x]^{\alpha} \subseteq[y]^{\alpha} \text {, for all } \alpha \in(0,1] \text {. }
$$

This order relation is given by the inclusion of subsets of $\mathbb{R}^{m}$.

Some interesting properties of fuzzy sets with respect to the partial orderings $\leq$ and $\preceq$ are

$$
\begin{aligned}
& x \leq y \Longrightarrow x+z \leq y+z, \quad \text { for } x, y, z \in E^{1}, \\
& x \preceq y \Longrightarrow x+z \preceq y+z, \quad \text { for } x, y, z \in E^{m},
\end{aligned}
$$

and, for fuzzy functions,

$$
\begin{array}{ll}
f \leq g \Longrightarrow \int_{0}^{t} f(s) d s \leq \int_{0}^{t} g(s) d s, & \text { for } t \in J, \quad f, g \in C\left(J, E^{1}\right), \\
f \preceq g \Longrightarrow \int_{0}^{t} f(s) d s \preceq \int_{0}^{t} g(s) d s, & \text { for } t \in J, \quad f, g \in C\left(J, E^{m}\right) .
\end{array}
$$

We will use them in sections 6 and 7 to obtain some existence results for fuzzy differential equations.

Reference [3] provides some results on existence of extreme fixed points for some kind of fuzzy mappings. Besides, in [3], the authors apply a Sadovskii-like fixed point theorem to solve the fuzzy equation $E x^{2}+F x+G=x$, with $E, F, G$, and $x$ nonnegative fuzzy numbers satisfying some appropriate conditions. In particular, in their approach they consider Lipschitzian fuzzy numbers.

In [15], the authors extend the results of [3] and some resolute results are deduced from the application of the classical Tarski's Fixed Point Theorem [22]. Also, it is considered in $E^{1}$, in relation with [16], the existence and uniqueness of solution for general fuzzy equations of the type

$$
F(x)=x
$$

In order to justify the validity of these results and deal with the case in $E^{m}, m>1$, we need to guarantee the validity of certain properties involving sequences of fuzzy sets and fuzzy functions. 


\section{Properties of spaces of fuzzy sets and fuzzy functions}

Firstly, we prove that the spaces $\left(E^{1}, \leq\right)$ and $\left(E^{1}, \preceq\right)$ satisfy conditions $(1)$ and $(2)$ (see Theorems 1.2 and 1.3). Indeed, consider $\left(x_{n}\right)_{n \in \mathbb{N}} \subseteq E^{1}$ a nondecreasing sequence such that $\left(x_{n}\right)_{n \in \mathbb{N}} \rightarrow x$ in $E^{1}$. Then the relation $x_{n} \leq x_{n+1}$ for every $n \in \mathbb{N}$ means that

$$
\left(x_{n}\right)_{\alpha l} \leq\left(x_{n+1}\right)_{\alpha l}, \quad\left(x_{n}\right)_{\alpha r} \leq\left(x_{n+1}\right)_{\alpha r}, \quad \forall \alpha \in(0,1], \forall n \in \mathbb{N} .
$$

Since

$$
\begin{aligned}
d_{\infty}\left(x_{n}, x\right) & =\sup _{\alpha \in[0,1]} d_{H}\left(\left[x_{n}\right]^{\alpha},[x]^{\alpha}\right) \\
& =\sup _{\alpha \in[0,1]} \max \left\{\left|\left(x_{n}\right)_{\alpha l}-x_{\alpha l}\right|,\left|\left(x_{n}\right)_{\alpha r}-x_{\alpha r}\right|\right\} \stackrel{n \rightarrow+\infty}{\longrightarrow} 0,
\end{aligned}
$$

then $\left(x_{n}\right)_{\alpha l} \rightarrow x_{\alpha l},\left(x_{n}\right)_{\alpha r} \rightarrow x_{\alpha r}$ as $n \rightarrow+\infty$, for every $\alpha \in(0,1]$, and they are nondecreasing sequences, which implies

$$
\left(x_{n}\right)_{\alpha l} \leq x_{\alpha l}, \quad\left(x_{n}\right)_{\alpha r} \leq x_{\alpha r} \quad \text { for every } n \text { and every } \alpha .
$$

Hence, $x_{n} \leq x, \forall n \in \mathbb{N}$.

Analogous conclusion can be derived for the partial ordering $\preceq$, since $x_{n} \preceq x_{n+1}$ for every $n \in \mathbb{N}$ implies $\left[x_{n}\right]^{\alpha} \subseteq\left[x_{n+1}\right]^{\alpha}$, for every $n \in \mathbb{N}, \alpha \in(0,1]$. Therefore, for every $\alpha,\left(x_{n}\right)_{\alpha l}$ is nonincreasing in $n$ and $\left(x_{n}\right)_{\alpha r}$ is nondecreasing in $n$. The convergent character of those sequences towards $x_{\alpha l}$ and $x_{\alpha r}$, respectively, provides that

$$
\left(x_{n}\right)_{\alpha l} \geq x_{\alpha l}, \quad\left(x_{n}\right)_{\alpha r} \leq x_{\alpha r} \quad \text { for every } n \in \mathbb{N} \text { and every } \alpha \in(0,1] .
$$

In consequence $\left[x_{n}\right]^{\alpha} \subseteq[x]^{\alpha}$, for every $n \in \mathbb{N}$ and $\alpha \in(0,1]$, so that $x_{n} \preceq x, \forall n \in \mathbb{N}$.

Similarly, it is easy to check that condition (2) also holds for the partial orderings $\leq$ and $\preceq$.

Consider the space $C\left(J, E^{1}\right)=\left\{x: J \longrightarrow E^{1}: x\right.$ is continuous $\}$, where $J$ is a compact interval in $\mathbb{R}$ and define the partial orderings induced by $\leq$ and $\preceq$ :

$$
\begin{aligned}
& x, y \in C\left(J, E^{1}\right), \quad x \leq y \Longleftrightarrow x(t) \leq y(t), \forall t \in J, \\
& x, y \in C\left(J, E^{1}\right), \quad x \preceq y \Longleftrightarrow x(t) \preceq y(t), \forall t \in J .
\end{aligned}
$$

Considering the distance

$$
D(x, y)=\sup _{t \in J} d_{\infty}(x(t), y(t)), \quad x, y \in C\left(J, E^{1}\right),
$$

$C\left(J, E^{1}\right)$ is a complete metric space [5].

Conditions (1) and (2) are satisfied for the partially ordered spaces

$$
\left(C\left(J, E^{1}\right), \leq\right)
$$


and

$$
\left(C\left(J, E^{1}\right), \preceq\right) .
$$

Indeed, if $\left(x_{n}\right) \subseteq C\left(J, E^{1}\right)$ is $\leq$-nondecreasing and convergent to $x$ in $C\left(J, E^{1}\right)$, then

$$
D\left(x_{n}, x\right)=\sup _{t \in J} d_{\infty}\left(x_{n}(t), x(t)\right)=\sup _{t \in J} \sup _{\alpha \in[0,1]} d_{H}\left(\left[x_{n}(t)\right]^{\alpha},[x(t)]^{\alpha}\right) \stackrel{n \rightarrow+\infty}{\longrightarrow} 0 .
$$

Hence, for every $t \in J,\left(x_{n}(t)\right)$ is a $\leq$-nondecreasing sequence in $E^{1}$ and convergent to $x(t) \in E^{1}$. This proves that $x_{n}(t) \leq x(t)$, for every $n \in \mathbb{N}$ and every $t$, hence $x_{n} \leq x$, $\forall n \in \mathbb{N}$. We obtain the same conclusion for $\preceq$ and, similarly, for property (2).

To check that condition (1) is valid in $\left(E^{m}, \preceq\right)$, take $\left(x_{n}\right)_{n \in \mathbb{N}} \subseteq E^{m}$ a nondecreasing sequence such that $\left(x_{n}\right)_{n \in \mathbb{N}} \rightarrow x$ in $E^{m}$. Then $x_{n} \preceq x_{n+1}$ for every $n \in \mathbb{N}$, that is,

$$
\left[x_{n}\right]^{\alpha} \subseteq\left[x_{n+1}\right]^{\alpha}, \forall n \in \mathbb{N} \text {, and } \alpha \in(0,1] .
$$

Moreover

$$
d_{\infty}\left(x_{n}, x\right)=\sup _{\alpha \in[0,1]} d_{H}\left(\left[x_{n}\right]^{\alpha},[x]^{\alpha}\right) \stackrel{n \rightarrow+\infty}{\longrightarrow} 0 .
$$

Thus, for every $\alpha \in(0,1],\left(\left[x_{n}\right]^{\alpha}\right)_{n \in \mathbb{N}}$ is an expansive family of subsets of $\mathbb{R}^{m}$ which is convergent. Moreover, it converges to $\overline{\bigcup_{n \in \mathbb{N}}\left[x_{n}\right]^{\alpha}}$. The convergence of $\left[x_{n}\right]^{\alpha}$ towards $[x]^{\alpha}$, for every $\alpha \in(0,1]$ implies $\left[x_{n}\right]^{\alpha} \subseteq[x]^{\alpha}=\overline{\bigcup_{n \in \mathbb{N}}\left[x_{n}\right]^{\alpha}}, \forall n \in \mathbb{N}, \forall \alpha \in(0,1]$, and $x_{n} \preceq x, \forall n \in \mathbb{N}$.

To prove the validity of (2), if $\left(x_{n}\right)_{n \in \mathbb{N}} \subseteq E^{m}$ is a nonincreasing and convergent sequence, then, for every $\alpha \in(0,1],\left(\left[x_{n}\right]^{\alpha}\right)_{n \in \mathbb{N}}$ is a contractive family which is convergent, therefore its limit is $\bigcap_{n \in \mathbb{N}}\left[x_{n}\right]^{\alpha}$, and $\left(x_{n}\right) \rightarrow x$ produces $\left[x_{n}\right]^{\alpha} \rightarrow[x]^{\alpha}$, as $n \rightarrow+\infty$, for every $\alpha \in(0,1]$, thus $[x]^{\alpha}=\bigcap_{n \in \mathbb{N}}\left[x_{n}\right]^{\alpha} \subseteq\left[x_{n}\right]^{\alpha}, \forall n \in \mathbb{N}, \forall \alpha \in(0,1]$, which implies $x \preceq x_{n}, \forall n \in \mathbb{N}$.

Now consider the space $C\left(J, E^{m}\right)=\left\{x: J \rightarrow E^{m}: x\right.$ is continuous $\}$ with the partial ordering

$$
x, y \in C\left(J, E^{m}\right), \quad x \preceq y \Longleftrightarrow x(t) \preceq y(t), \forall t \in J .
$$

$\left(C\left(J, E^{m}\right), D\right)$ is a complete metric space with the metric given by

$$
D(x, y)=\sup _{t \in J} d_{\infty}(x(t), y(t)), \quad x, y \in C\left(J, E^{m}\right) .
$$

In the partially ordered set $\left(C\left(J, E^{m}\right), \preceq\right)$, conditions (1) and (2) hold, since they are satisfied in $E^{m}$ and we proceed, for every $t \in J$, as in the one-dimensional case.

Concerning uniqueness of fixed point for a fuzzy mapping, condition (3) is valid for $\left(E^{1}, \leq\right),\left(E^{m}, \preceq\right),(m \geq 1),\left(C\left(J, E^{1}\right), \leq\right)$, and $\left(C\left(J, E^{m}\right), \preceq\right),(m \geq 1)$, since, in these spaces, any two elements have an upper bound. 


\section{Fixed Point Results}

Theorem 4.1 ([15]). Let $F: E^{1} \longrightarrow E^{1}$ be monotone nondecreasing (or nonincreasing and continuous). Suppose that there exists $K \in[0,1)$ such that

$$
d_{\infty}(F(x), F(y)) \leq K d_{\infty}(x, y), \quad \forall x \geq y
$$

and that there exists $x_{0} \in E^{1}$ with $F\left(x_{0}\right) \geq x_{0}$ or $F\left(x_{0}\right) \leq x_{0}$. Then there exists exactly one solution for equation $F(x)=x$.

Remark 4.2. We are not imposing that $F$ is a contraction, but $F$ is 'contractive for any two comparable elements' (see [20]).

Theorem 4.3. Let $F: C\left(J, E^{1}\right) \longrightarrow C\left(J, E^{1}\right)$ be monotone nondecreasing (or nonincreasing and continuous). Suppose that there exists $K \in[0,1)$ such that

$$
D(F(\varphi), F(\psi)) \leq K D(\varphi, \psi), \quad \forall \varphi \geq \psi
$$

and that there exists $\varphi_{0} \in C\left(J, E^{1}\right)$ with either $F\left(\varphi_{0}\right) \geq \varphi_{0}$ or $F\left(\varphi_{0}\right) \leq \varphi_{0}$. Then there exists exactly one solution for equation $F(\varphi)=\varphi$.

For the case of $m$-dimensional fuzzy sets, with $m \geq 1$, we obtain

Theorem 4.4. Let $F: E^{m} \longrightarrow E^{m}$ be monotone nondecreasing (or nonincreasing and continuous). Suppose that there exists $K \in[0,1)$ such that

$$
d_{\infty}(F(x), F(y)) \leq K d_{\infty}(x, y), \quad \forall x \succeq y
$$

and that there exists $x_{0} \in E^{m}$ with $F\left(x_{0}\right) \succeq x_{0}$ or $F\left(x_{0}\right) \preceq x_{0}$. Then there exists exactly one solution for equation $F(x)=x$.

Theorem 4.5. Let $F: C\left(J, E^{m}\right) \longrightarrow C\left(J, E^{m}\right)$ be monotone nondecreasing (or nonincreasing and continuous). Suppose that there exists $K \in[0,1)$ such that

$$
D(F(\varphi), F(\psi)) \leq K D(\varphi, \psi), \quad \forall \varphi \succeq \psi
$$

and assume that there exists $\varphi_{0} \in C\left(J, E^{m}\right)$ with $F\left(\varphi_{0}\right) \succeq \varphi_{0}$ or $F\left(\varphi_{0}\right) \preceq \varphi_{0}$. Then there exists exactly one solution for equation $F(\varphi)=\varphi$.

These theorems are consequence of Theorems 1.2-1.4.

Remark 4.6. If $F$ is continuous and maps comparable elements into comparable elements, then the results in this section are valid. 


\section{Examples}

We adapt some examples of [16] to the case of fuzzy functions.

Example 5.1. Consider the complete metric space $\left(E^{1}, d_{\infty}\right)$ and define the partial order

$$
x \leq_{1} y \Longleftrightarrow\left\{\begin{array}{l}
\chi_{\{n\}}<x \leq y \leq \chi_{\{n+1\}}, \quad \text { for some } n \in \mathbb{Z}, \\
\text { or } \\
x=y .
\end{array}\right.
$$

We show that $\leq_{1}$ defines a partial ordering in $E^{1}$.

- The reflexivity is obvious from the definition of $\leq_{1}$.

- If $x \leq_{1} y, y \leq_{1} x$, then $x=y$, or

$$
\chi_{\{n\}}<x \leq y \leq \chi_{\{n+1\}}, \quad \chi_{\{p\}}<y \leq x \leq \chi_{\{p+1\}}, \quad \text { for some } n, p \in \mathbb{Z},
$$

thus the unique possibility is $n=p$ and $x=y$.

- Let $x \leq_{1} y, y \leq_{1} z$ and suppose that $x \neq y, y \neq z$. Then

$$
\chi_{\{n\}}<x \leq y \leq \chi_{\{n+1\}}, \quad \chi_{\{p\}}<y \leq z \leq \chi_{\{p+1\}}, \quad \text { for some } n, p \in \mathbb{Z} .
$$

Since $y \notin\left(\chi_{\{n+1\}}, \chi_{\{n+2\}}\right]$, then $\chi_{\{n\}}<y \leq z \leq \chi_{\{n+1\}}$, and therefore $\chi_{\{n\}}<x \leq z \leq \chi_{\{n+1\}}$, obtaining $x \leq_{1} z$.

Consider the fuzzy function $F: E^{1} \rightarrow E^{1}$,

$$
F(x):=\chi_{\left\{\frac{n}{2}\right\}}+\frac{1}{2} x, \quad \text { if } \chi_{\{n\}}<x \leq \chi_{\{n+1\}}, \quad n=0,1,2, \ldots
$$

and $F(x):=\frac{1}{2} x$, otherwise.

$F$ is not continuous at $\chi_{\{n\}}$, for $n=0,1,2, \ldots$, since

$$
F\left(\chi_{\{n\}}\right)=\chi_{\left\{\frac{n-1}{2}\right\}}+\frac{1}{2} \chi_{\{n\}}=\chi_{\left\{n-\frac{1}{2}\right\}},
$$

the same as the limit of $F(x)$ as $x$ tends to $\chi_{\{n\}}$, with $x \leq \chi_{\{n\}}$. However, if $\chi_{\{n\}}<x \leq \chi_{\{n+1\}}$, and $x$ tends to $\chi_{\{n\}}$, then

$$
F(x)=\chi_{\left\{\frac{n}{2}\right\}}+\frac{1}{2} x \rightarrow \chi_{\left\{\frac{n}{2}\right\}}+\chi_{\left\{\frac{n}{2}\right\}}=\chi_{\{n\}} .
$$

Moreover, if $x$ tends to $\chi_{\{n\}}$, through $x$ not comparable to $\chi_{\{n\}}$, then $F(x)=\frac{1}{2} x$ tends to $\chi_{\left\{\frac{n}{2}\right\}}$.

However, this function $F$ is monotone nondecreasing. Indeed, take $x \leq_{1} y$. 
- If $x=y$, then $F(x)=F(y)$, hence $F(x) \leq_{1} F(y)$.

- If $\chi_{\{n\}}<x \leq y \leq \chi_{\{n+1\}}$, for some $n=0,1,2, \ldots$, then

$$
\begin{aligned}
\chi_{\{n\}} & =\chi_{\left\{\frac{n}{2}\right\}}+\chi_{\left\{\frac{n}{2}\right\}}<\chi_{\left\{\frac{n}{2}\right\}}+\frac{1}{2} x=F(x) \\
& \leq F(y)=\chi_{\left\{\frac{n}{2}\right\}}+\frac{1}{2} y \leq \chi_{\left\{\frac{n}{2}\right\}}+\chi_{\left\{\frac{n+1}{2}\right\}}=\chi_{\left\{n+\frac{1}{2}\right\}} \leq \chi_{\{n+1\}} .
\end{aligned}
$$

- If $\chi_{\{n\}}<x \leq y \leq \chi_{\{n+1\}}$, for some $n=-1,-2, \ldots$, then $F(x)=\frac{1}{2} x \leq \frac{1}{2} y=$ $F(y)$. Moreover,

$$
\chi_{\left\{\frac{n}{2}\right\}}<\frac{1}{2} x \leq \frac{1}{2} y \leq \chi_{\left\{\frac{n+1}{2}\right\}},
$$

which implies

$$
\chi_{\{k\}}=\chi_{\left\{\frac{n}{2}\right\}}<\frac{1}{2} x \leq \frac{1}{2} y \leq \chi_{\left\{\frac{n+1}{2}\right\}}=\chi_{\left\{k+\frac{1}{2}\right\}} \leq \chi_{\{k+1\}}, \quad \text { if } n=2 k,
$$

and

$$
\chi_{\{k\}}<\chi_{\left\{k+\frac{1}{2}\right\}}=\chi_{\left\{\frac{2 k+1}{2}\right\}}<\frac{1}{2} x \leq \frac{1}{2} y \leq \chi_{\left\{\frac{2 k+2}{2}\right\}}=\chi_{\{k+1\}}, \quad \text { if } n=2 k+1 .
$$

This proves that $F(x) \leq_{1} F(y)$.

Moreover, if $x \geq_{1} y$, we obtain

- If $x=y, d_{\infty}(F(x), F(y)) \leq \frac{1}{2} d_{\infty}(x, y)$.

- If $\chi_{\{n\}}<y \leq x \leq \chi_{\{n+1\}}$, for some $n=0,1,2, \ldots$, then

$$
d_{\infty}(F(x), F(y))=d_{\infty}\left(\chi_{\left\{\frac{n}{2}\right\}}+\frac{1}{2} x, \chi_{\left\{\frac{n}{2}\right\}}+\frac{1}{2} y\right)=d_{\infty}\left(\frac{1}{2} x, \frac{1}{2} y\right)=\frac{1}{2} d_{\infty}(x, y) .
$$

- If $\chi_{\{n\}}<y \leq x \leq \chi_{\{n+1\}}$, for some negative $n$, then

$$
d_{\infty}(F(x), F(y))=d_{\infty}\left(\frac{1}{2} x, \frac{1}{2} y\right)=\frac{1}{2} d_{\infty}(x, y) .
$$

To the validity of (1), let $\left(x_{n}\right)$ be a nondecreasing sequence in $E^{1}$ such that $\left(x_{n}\right) \rightarrow x$ in $E^{1}$. Then one of the following assertions is valid:

- either $x_{n}=x$, for every $n$, which implies that $x_{n} \leq_{1} x$, for every $n$,

- or there are different terms in the sequence. In this case, there exists $p \in \mathbb{Z}$ such that

$$
\chi_{\{p\}}<x_{n} \leq x_{n+1} \leq \chi_{\{p+1\}}, \quad \text { for every } n \in \mathbb{N} .
$$


By the properties of $\leq, x_{n} \leq x$ for every $n \in \mathbb{N}$ and besides

$$
\chi_{\{p\}}<x_{n} \leq x \leq \chi_{\{p+1\}}, \quad \text { for every } n \in \mathbb{N} .
$$

In consequence, $x_{n} \leq_{1} x$, for every $n \in \mathbb{N}$.

Finally,

$$
\chi_{\{0\}} \geq F\left(\chi_{\left\{-\frac{1}{2}\right\}}\right)=\frac{1}{2} \chi_{\left\{-\frac{1}{2}\right\}}=\chi_{\left\{-\frac{1}{4}\right\}} \geq \chi_{\left\{-\frac{1}{2}\right\}}>\chi_{\{-1\}}
$$

and, hence, $F\left(\chi_{\left\{-\frac{1}{2}\right\}}\right) \geq_{1} \chi_{\left\{-\frac{1}{2}\right\}}$.

By Theorem 1.2, there exists at least a fixed point of $F$ in $E^{1}\left(\chi_{\{0\}}\right.$ is a fixed point.) The uniqueness hypothesis (3) is not valid, since given $\chi_{\{n\}}, \chi_{\{n+1\}}$, there exists neither upper nor lower bound. However, there is exactly one fixed point for $F$ in $E^{1}$, since

- $\frac{1}{2} x=x$ is equivalent to $x=\chi_{\{0\}}$.

- For $\chi_{\{n\}}<x \leq \chi_{\{n+1\}}, n=0,1,2, \ldots$, then $\chi_{\left\{\frac{n}{2}\right\}}+\frac{1}{2} x=x$, implies $x=\chi_{\{n\}}$, which is a contradiction.

Example 5.2. Consider the partial ordering

$$
x \leq_{2} y \Longleftrightarrow\left\{\begin{array}{l}
\chi_{\{0\}} \leq x \leq y \leq \chi_{\{1\}}, \\
\text { or } \\
\chi_{\{n\}}<x \leq y \leq \chi_{\{n+1\}}, \quad \text { for some } n \in \mathbb{N}, \\
\text { or } \\
x=y,
\end{array}\right.
$$

and define the fuzzy function $\tilde{F}:\left\{x \in E^{1}: x \geq \chi_{\{0\}}\right\} \longrightarrow\left\{x \in E^{1}: x \geq \chi_{\{0\}}\right\}$,

$$
\tilde{F}(x):= \begin{cases}\chi_{\left\{\frac{n}{2}\right\}}+\frac{1}{2} x, & \text { if } \chi_{\{n\}}<x \leq \chi_{\{n+1\}}, \quad n \in \mathbb{N}, \\ \frac{1}{2} x, & \text { otherwise. }\end{cases}
$$

The uniqueness hypothesis is not valid but, in this case, $\chi_{\{0\}}$ is the unique fixed point of $\tilde{F}$. In fact, if we consider the restriction of $\tilde{F}$ to $\left[\chi_{\{0\}}, \chi_{\{1\}}\right]$

$$
\tilde{F}_{1}:\left[\chi_{\{0\}}, \chi_{\{1\}}\right] \longrightarrow\left[\chi_{\{0\}}, \chi_{\{1\}}\right],
$$

then the uniqueness condition is valid for $\tilde{F}_{1}$ and $\tilde{F}_{1}(x)=\frac{1}{2} x$.

For $x \notin\left[\chi_{\{0\}}, \chi_{\{1\}}\right]$, we obtain

- either $\tilde{F}(x)=\frac{1}{2} x$, and the unique fixed point is $\chi_{\{0\}}$, which is different from $x$, 
- or $\tilde{F}(x)=\chi_{\left\{\frac{n}{2}\right\}}+\frac{1}{2} x\left(\chi_{\{n\}}<x \leq \chi_{\{n+1\}}, n \in \mathbb{N}\right)$, and $\tilde{F}(y)=y$ occurs only for $y=\chi_{\{n\}} \notin\left(\chi_{\{n\}}, \chi_{\{n+1\}}\right]$.

Example 5.3. In the complete metric space $\left(E^{1}, d_{\infty}\right)$ take the partial ordering

$$
x \leq_{3} y \Longleftrightarrow\left\{\begin{array}{l}
\chi_{\{n\}} \leq x \leq y<\chi_{\{n+1\}}, \quad \text { for some } n=0,1,2, \ldots, \\
\text { or } \\
x=y .
\end{array}\right.
$$

Indeed,

- $\leq_{3}$ satisfies the reflexivity property.

- If $x \leq_{3} y, y \leq_{3} x$, then $x=y$ or

$$
\chi_{\{n\}} \leq x \leq y<\chi_{\{n+1\}}, \quad \chi_{\{n\}} \leq y \leq x<\chi_{\{n+1\}}, \quad \text { for some } n=0,1, \ldots,
$$

hence $x=y$.

- Let $x \leq_{3} y, y \leq_{3} z$ and suppose that $x \neq y, y \neq z$. Then

$$
\chi_{\{n\}} \leq x \leq y<\chi_{\{n+1\}}, \quad \chi_{\{n\}} \leq y \leq z<\chi_{\{n+1\}}, \quad \text { for some } n=0,1, \ldots
$$

In consequence, $\chi_{\{n\}} \leq x \leq z<\chi_{\{n+1\}}$, and $x \leq_{3} z$.

We define $F:\left\{x \in E^{1}: x \geq \chi_{\{0\}}\right\} \longrightarrow\left\{x \in E^{1}: x \geq \chi_{\{0\}}\right\}$, by

$$
F(x):= \begin{cases}\chi_{\left\{\frac{n+1}{2}\right\}}+\frac{1}{2} x, & \text { if } \chi_{\{n\}} \leq x<\chi_{\{n+1\}}, \quad n \in \mathbb{N}, \\ \frac{1}{2} x, & \text { otherwise. }\end{cases}
$$

It is evident that $F$ is not continuous at $\chi_{\{n\}}$, for $n \in \mathbb{N}$. On the other hand, $F$ is monotone nondecreasing. Indeed, let $x \leq_{3} y$.

- For $x=y, F(x)=F(y)$ and $F(x) \leq_{3} F(y)$.

- Suppose that $\chi_{\{n\}} \leq x \leq y<\chi_{\{n+1\}}$, for some $n=0,1,2, \ldots$ If $n=0$, then $\chi_{\{0\}} \leq F(x)=\frac{1}{2} x \leq \frac{1}{2} y=F(y)<\frac{1}{2} \chi_{\{1\}}<\chi_{\{1\}}$ and $F(x) \leq_{3} F(y)$. If $n \in \mathbb{N}$,

$$
\begin{aligned}
\chi_{\{n\}} & \leq \chi_{\left\{n+\frac{1}{2}\right\}}=\chi_{\left\{\frac{n+1}{2}\right\}}+\chi_{\left\{\frac{n}{2}\right\}} \leq \chi_{\left\{\frac{n+1}{2}\right\}}+\frac{1}{2} x=F(x) \\
& \leq F(y)=\chi_{\left\{\frac{n+1}{2}\right\}}+\frac{1}{2} y<\chi_{\left\{\frac{n+1}{2}\right\}}+\chi_{\left\{\frac{n+1}{2}\right\}}=\chi_{\{n+1\}} .
\end{aligned}
$$

Hence, $F(x) \leq_{3} F(y)$.

If $x \geq_{3} y$, then 
- If $x=y, d_{\infty}(F(x), F(y))=0 \leq \frac{1}{2} d_{\infty}(x, y)$.

- If $\chi_{\{0\}} \leq y \leq x<\chi_{\{1\}}$,

$$
d_{\infty}(F(x), F(y))=d_{\infty}\left(\frac{1}{2} x, \frac{1}{2} y\right)=\frac{1}{2} d_{\infty}(x, y) .
$$

- If $\chi_{\{n\}} \leq y \leq x<\chi_{\{n+1\}}$, for some $n=1,2, \ldots$, then

$$
d_{\infty}(F(x), F(y))=d_{\infty}\left(\chi_{\left\{\frac{n+1}{2}\right\}}+\frac{1}{2} x, \chi_{\left\{\frac{n+1}{2}\right\}}+\frac{1}{2} y\right)=\frac{1}{2} d_{\infty}(x, y) .
$$

This proves that the contractivity condition over comparable elements holds. The uniqueness hypothesis (3) is not valid, since given $\chi_{\{n\}}, \chi_{\{n+1\}}$, there exists neither upper nor lower bound. Condition (1) is not valid in the interval $\left[\chi_{\{n\}}, \chi_{\{n+1\}}\right)$, $(n=0,1,2, \ldots) . \quad F$ has the unique fixed point $\chi_{\{0\}}$, since, for $x \notin\left[\chi_{\{0\}}, \chi_{\{1\}}\right]$, $\frac{1}{2} x \neq x$, and for $x \in\left[\chi_{\{n\}}, \chi_{\{n+1\}}\right)$, with $n \in \mathbb{N}, F(x)=\chi_{\left\{\frac{n+1}{2}\right\}}+\frac{1}{2} x \neq x$.

Example 5.4. Consider the complete metric space $\left(E^{1}, d_{\infty}\right)$ and the partial order

$$
x \preceq_{1} y \Longleftrightarrow\left\{\begin{array}{l}
x \preceq y \preceq \chi_{[n, n+1]}, \quad \text { for some } n \in \mathbb{Z}, \text { with } x \neq \chi_{\{n\}}, \\
\text { or } \\
x=y .
\end{array}\right.
$$

The relation $\preceq_{1}$ defines a partial ordering in $E^{1}$ :

- The reflexivity is obvious from the definition of $\preceq_{1}$.

- If $x \preceq_{1} y, y \preceq_{1} x$, then $x=y$ or

$$
x \preceq y \preceq \chi_{[n, n+1]}, \quad y \preceq x \preceq \chi_{[p, p+1]}, \quad \text { for some } n, p \in \mathbb{Z},
$$

then $n=p$ and $x=y$.

- Let $x \preceq_{1} y, y \preceq_{1} z$ and suppose that $x \neq y, y \neq z$. Then $x \preceq y \preceq \chi_{[n, n+1]}$ and $y \preceq z \preceq \chi_{[n, n+1]}$, for some $n \in \mathbb{Z}$, with $x, y \neq \chi_{\{n\}}$. Then

$$
\chi_{\{n\}} \neq x \preceq z \preceq \chi_{[n, n+1]},
$$

and $x \preceq_{1} z$.

Define $F: E^{1} \rightarrow E^{1}$ by

$$
F(x):=\chi_{\left\{\frac{n}{2}\right\}}+\frac{1}{2} x, \quad \text { if } x \preceq \chi_{[n, n+1]}, \quad x \neq \chi_{\{n\}}, \quad n=0,1,2, \ldots
$$


and $F(x):=\frac{1}{2} x$, otherwise. $F$ is not continuous at $\chi_{\{n\}}$, for $n=1,2, \ldots$, since for $x \rightarrow \chi_{\{n\}}$, with $x \preceq \chi_{[n, n+1]}$, we obtain

$$
F(x)=\chi_{\left\{\frac{n}{2}\right\}}+\frac{1}{2} x \rightarrow \chi_{\left\{\frac{n}{2}\right\}}+\chi_{\left\{\frac{n}{2}\right\}}=\chi_{\{n\}},
$$

but for $x \rightarrow \chi_{\{n\}}$, with $x \preceq \chi_{[n-1, n]}$, we get

$$
F(x)=\chi_{\left\{\frac{n-1}{2}\right\}}+\frac{1}{2} x \rightarrow \chi_{\left\{\frac{n-1}{2}\right\}}+\chi_{\left\{\frac{n}{2}\right\}}=\chi_{\left\{n-\frac{1}{2}\right\}} .
$$

Besides, $F$ is monotone nondecreasing. Let $x \preceq_{1} y$. If $x=y$, then $F(x)=F(y)$, and $F(x) \preceq_{1} F(y)$. If $\chi_{\{n\}} \neq x \preceq y \preceq \chi_{[n, n+1]}$, for some $n=0,1,2, \ldots$, then

$$
\begin{aligned}
\chi_{\{n\}} & =\chi_{\left\{\frac{n}{2}\right\}}+\chi_{\left\{\frac{n}{2}\right\}} \neq \chi_{\left\{\frac{n}{2}\right\}}+\frac{1}{2} x=F(x) \preceq F(y)=\chi_{\left\{\frac{n}{2}\right\}}+\frac{1}{2} y \\
& \preceq \chi_{\left\{\frac{n}{2}\right\}}+\frac{1}{2} \chi_{[n, n+1]}=\chi_{\left\{\frac{n}{2}\right\}}+\chi_{\left[\frac{n}{2}, \frac{n+1}{2}\right]}=\chi_{\left[n, n+\frac{1}{2}\right]} \preceq \chi_{[n, n+1]} .
\end{aligned}
$$

If $\chi_{\{n\}} \neq x \preceq y \preceq \chi_{[n, n+1]}$, for some $n=-1,-2, \ldots$, then

$$
F(x)=\frac{1}{2} x \preceq \frac{1}{2} y=F(y) \preceq \frac{1}{2} \chi_{[n, n+1]}=\chi_{\left[\frac{n}{2}, \frac{n}{2}+\frac{1}{2}\right]} .
$$

Now, we distinguish two cases:

- If $n=2 k$, then

$$
\chi_{\left[\frac{n}{2}, \frac{n}{2}+\frac{1}{2}\right]}=\chi_{\left[k, k+\frac{1}{2}\right]} \preceq \chi_{[k, k+1]} .
$$

Since $x \neq \chi_{\{n\}}$, then $F(x)=\frac{1}{2} x \neq \chi_{\left\{\frac{n}{2}\right\}}=\chi_{\{k\}}$.

- If $n=2 k+1$, then

$$
\chi_{\left[\frac{n}{2}, \frac{n}{2}+\frac{1}{2}\right]}=\chi_{\left[k+\frac{1}{2}, k+1\right]} \preceq \chi_{[k, k+1]} .
$$

If $F(x)=\frac{1}{2} x=\chi_{\{k\}}$, then $x=\chi_{\{2 k\}}$ and $2 k<n$, contradicting $x \preceq \chi_{[n, n+1]}$.

This proves that $F(x) \preceq_{1} F(y)$.

With respect to the contractivity condition over comparable elements, take $x \succeq_{1} y$,

- If $x=y, d_{\infty}(F(x), F(y))=0 \leq \frac{1}{2} d_{\infty}(x, y)$.

- If $\chi_{\{n\}} \neq y \preceq x \preceq \chi_{[n, n+1]}$, for some $n=0,1,2, \ldots$, then

$$
d_{\infty}(F(x), F(y))=d_{\infty}\left(\chi_{\left\{\frac{n}{2}\right\}}+\frac{1}{2} x, \chi_{\left\{\frac{n}{2}\right\}}+\frac{1}{2} y\right)=\frac{1}{2} d_{\infty}(x, y) .
$$


- If $\chi_{\{n\}} \neq y \preceq x \preceq \chi_{[n, n+1]}$, for some negative $n$, then

$$
d_{\infty}(F(x), F(y))=d_{\infty}\left(\frac{1}{2} x, \frac{1}{2} y\right)=\frac{1}{2} d_{\infty}(x, y) .
$$

Note that $\chi_{\{0\}} \preceq_{1} F\left(\chi_{\{0\}}\right)$.

Finally, concerning condition (1), take $\left(x_{n}\right)$ a nondecreasing sequence in $E^{1}$ such that $\left(x_{n}\right) \rightarrow x$ in $E^{1}$, then one of the following assertions is valid:

- $x_{n}=x$, for every $n \in \mathbb{N}$, which implies that $x_{n} \preceq_{1} x$, for every $n \in \mathbb{N}$.

- There exists $p \in \mathbb{Z}$ such that

$$
\chi_{\{p\}} \neq x_{1} \preceq x_{2} \preceq \cdots \preceq x_{n} \preceq x_{n+1} \preceq \cdots \preceq \chi_{[p, p+1]} .
$$

In consequence, $\chi_{\{p\}} \neq x_{n} \preceq x \preceq \chi_{[p, p+1]}$, for every $n \in \mathbb{N}$, and $x_{n} \preceq_{1} x$, for every $n \in \mathbb{N}$.

Then $F$ has at least one fixed point in $E^{1}$. (Note that $\chi_{\{0\}}$ is a fixed point of $F$, in fact, it is the unique fixed point of $F$.)

\section{Fuzzy initial value problems}

In this section, we obtain new results on existence of a unique solution for a fuzzy differential equation with initial value conditions by applying the previous methodology. Consider the fuzzy equation

$$
\left\{\begin{array}{l}
u^{\prime}(t)=f(t, u(t)), \quad t \in J=[0, T], \\
u(0)=u_{0},
\end{array}\right.
$$

where $T>0, u_{0} \in E^{1}$, and $f: J \times E^{1} \longrightarrow E^{1}$ is a continuous function. This initial value problem has been considered in the literature by several authors. For example, Kaleva [9] proved that Picard's Theorem is valid for (5), i.e., when $f$ is Lipschitz continuous, then (5) has a unique (local) solution. If $f$ is just continuous, the situation is totally different and some conditions are necessary to guarantee the existence of solution $[1,2,4,6,8-10,13,14,19,21,23,24]$. Also, new phenomena appear in the fuzzy case $[4,5,8,17]$.

Let the space $C^{1}\left(J, E^{1}\right)=\left\{x: J \longrightarrow E^{1}: x, x^{\prime}\right.$ are continuous $\}$, where the derivative $x^{\prime}$ of $x$ is considered in the sense of Hukuhara.

Definition 6.1. A solution to (5) is a function $u \in C^{1}\left(J, E^{1}\right)$ satisfying (5).

Definition 6.2. A lower solution for (5) is a function $\mu \in C^{1}\left(J, E^{1}\right)$ such that

$$
\mu^{\prime}(t) \leq f(t, \mu(t)), \quad t \in J, \quad \mu(0) \leq u_{0} .
$$

An upper solution for (5) satisfies the reversed inequalities. 
For the case of real functions, the basic reference concerning the method of upper and lower solutions is [11], which shows how the existence of a lower solution $\mu$ and an upper solution $\nu$ with $\mu \leq \nu$ implies the existence of a solution of (5) between $\mu$ and $\nu$.

In the following, we study the fuzzy case and find sufficient conditions for the existence of a unique solution to the first-order initial value problem (5) in the presence of just a lower solution (respectively, an upper solution). We follow the ideas of [16] and [18], where we showed that, under the appropriate conditions, the existence of just a lower solution is enough to prove the existence of a unique solution for the following (nonfuzzy) periodic boundary value problem:

$$
\left\{\begin{array}{l}
u^{\prime}(t)=f(t, u(t)), \quad t \in J=[0, T], \\
u(0)=u(T),
\end{array}\right.
$$

where $T>0$, and $f: J \times \mathbb{R} \longrightarrow \mathbb{R}$.

Theorem 6.3. Suppose that there exists $\mu \in C^{1}\left(J, E^{1}\right)$ a lower solution for problem (5). Let $f: J \times E^{1} \longrightarrow E^{1}$ continuous be such that $f$ is nondecreasing in the second variable

$$
f(t, x) \geq f(t, y), \quad \text { if } \quad t \in J, \quad x \geq y,
$$

and there exists $k \geq 0$ such that

$$
d_{\infty}(f(t, x), f(t, y)) \leq k d_{\infty}(x, y), \quad x \geq y .
$$

Then problem (5) has a unique solution.

Proof. Problem (5) is written, equivalently $[5,12,17]$, as the integral equation

$$
u(t)=u_{0}+\int_{0}^{t} f(s, u(s)) d s, \quad t \in J .
$$

We define the operator $\mathcal{A}: C\left(J, E^{1}\right) \longrightarrow C\left(J, E^{1}\right)$ by

$$
[\mathcal{A} u](t)=u_{0}+\int_{0}^{t} f(s, u(s)) d s, \quad t \in J .
$$

If $u \in C\left(J, E^{1}\right)$ is a fixed point of $\mathcal{A}$, then $u \in C^{1}\left(J, E^{1}\right)$ is a solution of (5) and conversely. In $C\left(J, E^{1}\right)$, for $\rho>0$, consider the complete metric

$$
D_{\rho}(u, v)=\sup _{t \in J}\left\{d_{\infty}(u(t), v(t)) \cdot e^{-\rho t}\right\}, \quad u, v \in C\left(J, E^{1}\right) .
$$

This metric is equivalent to $D$. Here $\rho>0$ is chosen large enough so that

$$
k \frac{1-e^{-\rho T}}{\rho}<1 .
$$


Hypotheses in Theorem 1.2 and condition (3) are satisfied. Indeed, condition (1) is valid. The operator $\mathcal{A}$ is nondecreasing since, for $u \geq v$,

$$
[\mathcal{A} u](t)=u_{0}+\int_{0}^{t} f(s, u(s)) d s \geq u_{0}+\int_{0}^{t} f(s, v(s)) d s=[\mathcal{A} v](t), \quad t \in J .
$$

Besides, for $u \geq v$,

$$
\begin{aligned}
D_{\rho}(\mathcal{A} u, \mathcal{A} v) & =\sup _{t \in J}\left\{d_{\infty}([\mathcal{A} u](t),[\mathcal{A} v](t)) e^{-\rho t}\right\} \\
& =\sup _{t \in J}\left\{d_{\infty}\left(u_{0}+\int_{0}^{t} f(s, u(s)) d s, u_{0}+\int_{0}^{t} f(s, v(s)) d s\right) e^{-\rho t}\right\} \\
& =\sup _{t \in J}\left\{d_{\infty}\left(\int_{0}^{t} f(s, u(s)) d s, \int_{0}^{t} f(s, v(s)) d s\right) e^{-\rho t}\right\} \\
& \leq \sup _{t \in J}\left\{\int_{0}^{t} d_{\infty}(f(s, u(s)), f(s, v(s))) d s e^{-\rho t}\right\} \\
& \leq \sup _{t \in J}\left\{\int_{0}^{t} k d_{\infty}(u(s), v(s)) d s e^{-\rho t}\right\} \leq D_{\rho}(u, v) k \sup _{t \in J}\left\{\frac{e^{\rho t}-1}{\rho} e^{-\rho t}\right\} \\
& =D_{\rho}(u, v) k \sup _{t \in J} \frac{1-e^{-\rho t}}{\rho}=D_{\rho}(u, v) k \frac{1-e^{-\rho T}}{\rho},
\end{aligned}
$$

and the contractivity condition in Theorem 1.2 is satisfied since (6) holds.

Finally, we check that $\mu$ is such that $\mu \leq \mathcal{A} \mu$. Indeed,

$$
\mu(t)=\mu(0)+\int_{0}^{t} \mu^{\prime}(s) d s \leq u_{0}+\int_{0}^{t} f(s, \mu(s)) d s=[\mathcal{A} \mu](t), \quad t \in J .
$$

In consequence, $\mathcal{A}$ has a fixed point in $C\left(J, E^{1}\right)$. The uniqueness comes from the validity of (3).

Remark 6.4. The unique solution of (5) given by Theorem 6.3 can be obtained as $\lim _{n \rightarrow+\infty} \mathcal{A}^{n}(u)$, for any $u \in C\left(J, E^{1}\right)$. Taking $u=\mu$, then $\left(\mathcal{A}^{n}(\mu)\right)_{n}$ is a monotone nondecreasing sequence converging in $C\left(J, E^{1}\right)$ to the unique solution of (5).

Theorem 6.5. Replacing the existence of a lower solution to (5) by the existence of an upper solution to problem (5), the conclusion of Theorem 6.3 is still valid.

Proof. If $\nu$ is an upper solution for (5), we check that

$$
\nu(t)=\nu(0)+\int_{0}^{t} \nu^{\prime}(s) d s \geq u_{0}+\int_{0}^{t} f(s, \nu(s)) d s=[\mathcal{A} \nu](t), \quad t \in J .
$$

The existence of a unique solution for (5) is derived from Theorem 1.3, since (2) is valid. 
For the case of $m$-dimensional fuzzy functions $f: J \times E^{m} \longrightarrow E^{m},(m \geq 1)$, we obtain similar results, considering the partial ordering $\preceq$. Let the fuzzy equation

$$
\left\{\begin{array}{l}
u^{\prime}(t)=f(t, u(t)), \quad t \in J=[0, T] \\
u(0)=u_{0},
\end{array}\right.
$$

where $T>0, u_{0} \in E^{m}$, and $f: J \times E^{m} \longrightarrow E^{m}$ is continuous. Take

$$
C^{1}\left(J, E^{m}\right)=\left\{x: J \longrightarrow E^{m}: x, x^{\prime} \text { continuous }\right\} .
$$

Definition 6.6. A solution to (7) is a function $u \in C^{1}\left(J, E^{m}\right)$ satisfying conditions in (7).

Definition 6.7. A lower solution for (7) is a function $\mu \in C^{1}\left(J, E^{m}\right)$ such that

$$
\mu^{\prime}(t) \preceq f(t, \mu(t)), \quad t \in J, \quad \mu(0) \preceq u_{0} .
$$

An upper solution for (7) is a function satisfying the reversed inequalities.

Theorem 6.8. Suppose that there exists $\mu \in C^{1}\left(J, E^{m}\right)$ a lower solution for problem (7). Let $f: J \times E^{m} \longrightarrow E^{m}$ continuous be such that $f$ is nondecreasing in the second variable $f(t, x) \succeq f(t, y)$, if $t \in J, x \succeq y$, and there exists $k \geq 0$ such that

$$
d_{\infty}(f(t, x), f(t, y)) \leq k d_{\infty}(x, y), \quad x \succeq y .
$$

Then problem (7) has a unique solution.

Proof. Analogous to the proof of Theorem 6.3.

Remark 6.9. The unique solution of (7) given by Theorem 6.8 can be obtained as $\lim _{n \rightarrow+\infty} \mathcal{A}^{n}(u)$, for any $u \in C\left(J, E^{m}\right)$. If we take $u=\mu$, then $\left(\mathcal{A}^{n}(\mu)\right)_{n}$ is a monotone nondecreasing sequence converging in $C\left(J, E^{m}\right)$ to the unique solution of $(7)$.

Theorem 6.10. Replacing the existence of a lower solution to (7) by the existence of an upper solution to problem (7), the conclusion of Theorem 6.8 is still valid.

Proof. Similar to the proof of Theorem 6.5.

\section{Fuzzy functional problems}

For a given $\tau>0$, we denote $C_{0}=C\left([-\tau, 0], E^{m}\right)$. For elements $\varphi, \psi \in C_{0}$, we define

$$
H(\varphi, \psi)=\max _{-\tau \leq s \leq 0} d_{\infty}(\varphi(s), \psi(s)) .
$$

Suppose that $u \in C\left(J_{0}, E^{m}\right)$, where $J_{0}=[-\tau, T], T>0$. For $t \geq 0, t \in J_{0}$, denote by $u_{t}$ the translation to $[-\tau, 0]$ of the restriction of $u$ to the interval $[t-\tau, t]$, that is, $u_{t} \in C_{0}, u_{t}(s)=u(t+s),-\tau \leq s \leq 0$. 
We consider the fuzzy differential equation with finite delay

$$
\left\{\begin{array}{l}
u^{\prime}(t)=f\left(t, u_{t}\right), \quad t \in J=[0, T], \\
u_{0}=\varphi \in C_{0}
\end{array}\right.
$$

where $f \in C\left(J \times C_{0}, E^{m}\right)$.

In [12], it is proved the following result.

Theorem 7.1. Assume that there exists $k>0$ such that

$$
d_{\infty}(f(t, \varphi), f(t, \psi)) \leq k H(\varphi, \psi), \text { for } t \in J=[0, T] \text { and } \varphi, \psi \in C_{0} .
$$

Then the initial value problem (8) possesses a unique solution $u(t)$ on $J_{0}$.

In this section, we relax the hypothesis of contractivity affecting only to comparable elements, and still we obtain the existence of a unique solution. To this purpose, we define the concepts of upper solution and lower solution for (8).

Definition 7.2. A solution to (8) is a function $u \in C\left(J_{0}, E^{m}\right) \cap C^{1}\left(J, E^{m}\right)$ satisfying (8).

Definition 7.3. A lower solution for (8) is a function $\mu \in C\left(J_{0}, E^{m}\right) \cap C^{1}\left(J, E^{m}\right)$ such that

$$
\mu^{\prime}(t) \preceq f\left(t, \mu_{t}\right), \quad t \in J, \quad \mu_{0} \preceq \varphi .
$$

(Also $\mu^{\prime}(t) \leq f\left(t, \mu_{t}\right), t \in J, \mu_{0} \leq \varphi$, if $m=1$.) An upper solution for (8) satisfies the reversed inequalities.

Theorem 7.4. Suppose that there exists $\mu \in C\left(J_{0}, E^{m}\right) \cap C^{1}\left(J, E^{m}\right)$ a lower solution for problem (8). Let $f: J \times C_{0} \longrightarrow E^{m}$ be continuous such that $f$ is nondecreasing in the second variable

$$
f(t, \varphi) \succeq f(t, \psi), \quad \text { if } \quad t \in J, \quad \varphi \succeq \psi,
$$

and there exists $k \geq 0$ such that

$$
d_{\infty}(f(t, \varphi), f(t, \psi)) \leq k H(\varphi, \psi), \quad t \in J, \quad \varphi \succeq \psi .
$$

Then problem (8) has a unique solution.

Proof. Consider the space $C\left(J_{0}, E^{m}\right)$ equipped with the complete metric

$$
D_{\rho}(u, v)=\sup _{t \in[-\tau, T]}\left\{d_{\infty}(u(t), v(t)) \cdot e^{-\rho t}\right\}, \quad u, v \in C\left(J_{0}, E^{m}\right),
$$

for $\rho>0$. 
We define the operator $\mathcal{A}: C\left(J_{0}, E^{m}\right) \longrightarrow C\left(J_{0}, E^{m}\right)$ by

$$
[\mathcal{A} u](t)= \begin{cases}\varphi(t), & t \in[-\tau, 0], \\ \varphi(0)+\int_{0}^{t} f\left(s, u_{s}\right) d s, & t \in J .\end{cases}
$$

If $u \in C\left(J_{0}, E^{m}\right)$ is a fixed point of $\mathcal{A}$, then $u \in C^{1}\left(J, E^{m}\right)$ is a solution of (8) and conversely. We check that conditions in Theorem 1.2 and condition (3) are satisfied. The mapping $\mathcal{A}$ is nondecreasing. Indeed, take $u \succeq v$ on $J_{0}\left(u_{s} \succeq v_{s}, \forall s \in J\right)$ and $t \in[-\tau, 0]$, then $(\mathcal{A} u)(t)=\varphi(t)=(\mathcal{A} v)(t)$, and, for $t \in J$,

$$
[\mathcal{A} u](t)=\varphi(0)+\int_{0}^{t} f\left(s, u_{s}\right) d s \succeq \varphi(0)+\int_{0}^{t} f\left(s, v_{s}\right) d s=[\mathcal{A} v](t) .
$$

For $u \preceq v$, if $t \in[-\tau, 0], d_{\infty}([\mathcal{A} u](t),[\mathcal{A} v](t))=d_{\infty}(\varphi(t), \varphi(t))=0$, and if $t \in[0, T]$,

$$
\begin{aligned}
d_{\infty}([\mathcal{A} u](t),[\mathcal{A} v](t)) & =d_{\infty}\left(\varphi(0)+\int_{0}^{t} f\left(s, u_{s}\right) d s, \varphi(0)+\int_{0}^{t} f\left(s, v_{s}\right) d s\right) \\
& \leq \int_{0}^{t} d_{\infty}\left(f\left(s, u_{s}\right), f\left(s, v_{s}\right)\right) d s \\
& \leq \int_{0}^{t} k H\left(u_{s}, v_{s}\right) d s=k \int_{0}^{t} \max _{-\tau \leq r \leq 0} d_{\infty}\left(u_{s}(r), v_{s}(r)\right) d s,
\end{aligned}
$$

then

$$
\begin{aligned}
D_{\rho}(\mathcal{A} u, \mathcal{A} v) & =\sup _{t \in[-\tau, T]}\left\{d_{\infty}([\mathcal{A} u](t),[\mathcal{A} v](t)) e^{-\rho t}\right\} \\
& \leq \sup _{t \in J}\left\{k \int_{0}^{t} \max _{-\tau \leq r \leq 0} d_{\infty}\left(u_{s}(r), v_{s}(r)\right) d s e^{-\rho t}\right\} \\
& \leq \sup _{t \in J}\left\{k \int_{0}^{t} \max _{-\tau \leq r \leq 0} e^{\rho(s+r)} d s e^{-\rho t}\right\} D_{\rho}(u, v) \\
& \leq \sup _{t \in J}\left\{k \int_{0}^{t} e^{\rho s} d s e^{-\rho t}\right\} D_{\rho}(u, v) \\
& =k D_{\rho}(u, v) \sup _{t \in J} \frac{1-e^{-\rho t}}{\rho}=k \frac{1-e^{-\rho T}}{\rho} D_{\rho}(u, v) .
\end{aligned}
$$

Note that $k=0$ is a trivial case. If we take $\rho=2 k$ (for $k>0$ ), the contractivity condition in Theorem 1.2 holds.

Finally, we check that $\mu$ is such that $\mu \preceq \mathcal{A} \mu$. Indeed, $\mu_{0} \preceq \varphi$, thus $\mu(t) \preceq \varphi(t)=$ $[\mathcal{A} \mu](t)$, for $t \in[-\tau, 0]$, and for $t \in J$,

$$
\mu(t)=\mu(0)+\int_{0}^{t} \mu^{\prime}(s) d s \preceq \varphi(0)+\int_{0}^{t} f\left(s, \mu_{s}\right) d s=[\mathcal{A} \mu](t) .
$$


Since every pair of functions in $C\left(J_{0}, E^{m}\right)$ has an upper bound, the mapping $\mathcal{A}$ has a unique fixed point $u \in C\left(J_{0}, E^{m}\right)$ and $u$ is the unique solution to the initial value problem (8) in $J_{0}$.

Theorem 7.5. Replacing the existence of a lower solution for (8) by the existence of an upper solution for problem (8), the conclusion of Theorem 7.4 is still valid.

Proof. If $\nu$ is an upper solution for (8), we check that

$$
\begin{gathered}
\nu(t) \succeq \varphi(t)=[\mathcal{A} \nu](t), t \in[-\tau, 0], \\
\nu(t)=\nu(0)+\int_{0}^{t} \nu^{\prime}(s) d s \succeq \varphi(0)+\int_{0}^{t} f\left(s, \nu_{s}\right) d s=[\mathcal{A} \nu](t), \quad t \in J .
\end{gathered}
$$

The existence of a unique solution for (8) comes from Theorem 1.3, since (2) is valid.

Remark 7.6. Under the hypotheses of the previous results, the unique solution of (8) is obtained as $\lim _{n \rightarrow+\infty} \mathcal{A}^{n}(u)$, for any $u \in C\left(J_{0}, E^{m}\right)$. If we take $u=\mu$, a lower solution for $(8)$, then $\left(\mathcal{A}^{n}(\mu)\right)_{n}$ is a monotone nondecreasing sequence converging in $C\left(J_{0}, E^{m}\right)$ to the unique solution of (8) and if $u=\nu$, an upper solution for (8), then $\left(\mathcal{A}^{n}(\nu)\right)_{n}$ is a monotone nonincreasing sequence which is convergent to the unique solution of $(8)$.

The results in this section are valid for $E^{1}$ with the partial ordering $\leq$.

Theorem 7.7. Suppose that there exists $\mu \in C\left(J_{0}, E^{1}\right) \cap C^{1}\left(J, E^{1}\right)$ a lower solution for problem (8). Let $f: J \times C_{0} \longrightarrow E^{1}$ continuous be such that $f$ is nondecreasing in the second variable

$$
f(t, \varphi) \geq f(t, \psi), \quad \text { if } \quad t \in J, \quad \varphi \geq \psi
$$

and there exists $k \geq 0$ such that

$$
d_{\infty}(f(t, \varphi), f(t, \psi)) \leq k H(\varphi, \psi), \quad t \in J, \quad \varphi \geq \psi
$$

Then problem (8) has a unique solution.

Theorem 7.8. Replacing the existence of a lower solution for (8) by the existence of an upper solution for problem (8), the conclusion of Theorem 7.7 is still valid.

Remark 7.9. The validity of (1) and (2) in fuzzy spaces allows to consider fuzzy differential equations with discontinuous nonlinearities. 


\section{References}

[1] S. Abbasbandy, J. J. Nieto, and M. Alavi, Tuning of reachable set in one dimensional fuzzy differential inclusions, Chaos Solitons Fractals 26 (2005), no. 5, 1337-1341.

[2] T. G. Bhaskar, V. Lakshmikantham, and V. Devi, Revisiting fuzzy differential equations, Nonlinear Anal. 58 (2004), no. 3-4, 351-358.

[3] J.-C. Chang, H. Chen, S.-M. Shyu, and W.-C. Lian, Fixed-point theorems in fuzzy real line, Comput. Math. Appl. 47 (2004), no. 6-7, 845-851.

[4] P. Diamond, Brief note on the variation of constants formula for fuzzy differential equations, Fuzzy Sets and Systems 129 (2002), no. 1, 65-71.

[5] P. Diamond and P. E. Kloeden, Metric spaces of fuzzy sets: theory and applications, World Scientific Publishing Co. Inc., River Edge, NJ, 1994.

[6] D. N. Georgiou, J. J. Nieto, and R. Rodríguez-López, Initial value problems for higher-order fuzzy differential equations, Nonlinear Anal. 63 (2005), no. 4, 587-600.

[7] S. Heikkilä and V. Lakshmikantham, Monotone iterative techniques for discontinuous nonlinear differential equations, Monographs and Textbooks in Pure and Applied Mathematics, vol. 181, Marcel Dekker Inc., New York, 1994.

[8] E. Hüllermeier, An approach to modelling and simulation of uncertain dynamical systems, Internat. J. Uncertain. Fuzziness Knowledge-Based Systems 5 (1997), no. 2, 117-137.

[9] O. Kaleva, Fuzzy differential equations, Fuzzy Sets and Systems 24 (1987), no. 3, 301-317.

[10] _ The Cauchy problem for fuzzy differential equations, Fuzzy Sets and Systems 35 (1990), no. $3,389-396$.

[11] G. S. Ladde, V. Lakshmikantham, and A. S. Vatsala, Monotone iterative techniques for nonlinear differential equations, Monographs, Advanced Texts and Surveys in Pure and Applied Mathematics, vol. 27, Pitman, Boston, MA, 1985.

[12] V. Lakshmikantham and R. N. Mohapatra, Theory of fuzzy differential equations and inclusions, Series in Mathematical Analysis and Applications, vol. 6, Taylor \& Francis Ltd., London, 2003.

[13] V. Lakshmikantham and J. J. Nieto, Differential equations in metric spaces: an introduction and an application to fuzzy differential equations, Dyn. Contin. Discrete Impuls. Syst. Ser. A Math. Anal. 10 (2003), no. 6, 991-1000.

[14] J. J. Nieto, The Cauchy problem for continuous fuzzy differential equations, Fuzzy Sets and Systems 102 (1999), no. 2, 259-262.

[15] J. J. Nieto and R. Rodríguez-López, Existence of extremal solutions for quadratic fuzzy equations, Fixed Point Theory Appl. (2005), no. 3, 321-342.

[16] _ Contractive mapping theorems in partially ordered sets and applications to ordinary differential equations, Order 22 (2005), no. 3, 223-239.

[17] _ Bounded solutions for fuzzy differential and integral equations, Chaos Solitons Fractals 27 (2006), no. 5, 1376-1386.

[18] _ Existence and uniqueness of fixed point in partially ordered sets and applications to ordinary differential equations, Acta Math. Sinica (Engl. Ser.), to appear.

[19] D. O'Regan, V. Lakshmikantham, and J. J. Nieto, Initial and boundary value problems for fuzzy differential equations, Nonlinear Anal. 54 (2003), no. 3, 405-415.

[20] A. C. M. Ran and M. C. B. Reurings, A fixed point theorem in partially ordered sets and some applications to matrix equations, Proc. Amer. Math. Soc. 132 (2004), no. 5, 1435-1443.

[21] S. Seikkala, On the fuzzy initial value problem, Fuzzy Sets and Systems 24 (1987), no. 3, 319330 . 
[22] A. Tarski, A lattice-theoretical fixpoint theorem and its applications, Pacific J. Math. 5 (1955), 285-309.

[23] D. Vorobiev and S. Seikkala, Towards the theory of fuzzy differential equations, Fuzzy Sets and Systems 125 (2002), no. 2, 231-237.

[24] C. Wu, S. Song, and E. S. Lee, Approximate solutions, existence, and uniqueness of the Cauchy problem of fuzzy differential equations, J. Math. Anal. Appl. 202 (1996), no. 2, 629-644. 